Mitteilungen der Österreichischen Geographischen Gesellschaft,

151. Jg. (Jahresband), Wien 2009, S. 291-303

\title{
LERNPSYCHOLOGISCHE UND MEDIALE GRUNDLAGEN im Hinblick aUf Kartographische InformationsSysteme als Geographie-Lehrmittel
}

\author{
Beatrix BRUNNER-FRIEDRICH, Mirjanka LeChTHALER \\ und Eszter SimonNÉ-DombóvÁRI, alle Wien*
}

mit 1 Abb. im Text

\section{INHALT}

Summary 291

Zusammenfassung

1 Einleitun 292

2 Wahrnehmungsverlauf und lernpsychologische Grundlagen 293

3 Einsatz interaktiver und multimedialer kartographischer

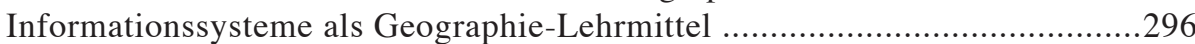

4 Anpassung Kartographischer Informationssysteme an die Benutzergruppen ...299

5 Fazit

6 Literaturverzeichnis 301

\section{Summary}

Psychological principles of learning and media basics with a view to cartographic information systems as tools for geographic education

Students as users of cartographic products have different abilities to acquire and process spatial information from cartographic presentations. They exhibit different learning styles and can therefore be classified in different types of learners. Today's teaching aids often address only some or just one of those learning types, whereas others are disregarded.

\footnotetext{
* Di Dr. Beatrix Brunner-Friedrich, Ass.-Prof. DI Dr. Mirjanka Lechthaler, Univ.-Ass. Eszter SimonNÉ-DombóvÁRI MSc., alle Institut für Geoinformation und Kartographie, Forschungsgruppe Kartographie, Technische Universität Wien,Erzherzog-Johann-Platz 1, 1040 Wien; e-mail: beatrix_f@ yahoo.de, lechthaler@tuwien.ac.at, eszter@cartography.tuwien.ac.at; http://www.ikr.tuwien.ac.at
} 
The main target of this article is to present the application of psychological principles of learning and media basics in Cartographic Information Systems in their usage as tools for geographic education. Such teaching aids do not allow for a completely individualised information presentation. However, a user group specific transport of information can be achieved.

\section{Zusammenfassung}

Schüler als Benutzer kartographischer Produkte weisen unterschiedliche Fähigkeiten auf, um raumbezogene Informationen aus kartographischen Präsentationsformen zu erfassen und zu verarbeiten. Schüler haben unterschiedliche Lernstile und können in verschiedene Lerntypen unterteilt werden. Heutige Unterrichtsmittel sprechen häufig nur bestimmte Lerntypen an, während andere vernachlässigt werden.

Ziel dieser Präsentation ist, lernpsychologische und mediale Grundlagen vorzustellen, um diese bei der Anwendung Kartographischer Informationssysteme als Lehrmittel im Geographie- und Wirtschaftskunde-Unterricht zu nützen. Mit solchen Unterrichtshilfen kann zwar keine vollständige Individualisierung der Informationspräsentation, aber eine benutzergruppenangepasste Informationsvermittlung ermöglicht werden.

\section{Einleitung}

In den letzten Jahren hat sich die Verwendung digitaler Medien in Schulen wesentlich intensiviert. Die Maßnahmen der Behörden, möglichst alle Schulen mit Schülercomputern und Internetanschlüssen auszurüsten, fördern diesen Trend. Es entspricht ihm, geeignete Software zu entwickeln, die psychologischen, didaktischen und fachlichen Ansprüchen gerecht wird (BRUNNER-FRIEDRICH 2004b). Impulse dazu werden mit der vorliegenden Arbeit gegeben.

Grundlage ist die These, dass Menschen sowohl unterschiedlich wahrnehmen (unterschiedlichen Lerntypen angehören) als auch das Wahrgenommene unterschiedlich verarbeiten, also in verschiedenen Formen lernen (unterschiedliche Lernstile haben). Die Darbietung von Stoffinhalten soll daher in einer dem Lernenden angepassten Form erfolgen, um einen effizienten Unterrichtsertrag im kognitiven wie auch im instrumentalen Bereich zu erzielen.

Eine starre Klassifizierung in einzelne Lerntypen ist allerdings nicht ganz angebracht. Die meisten Menschen sind Mischtypen, die keine Bevorzugung aufweisen. Darüber hinaus kann ein Individuum bei verschiedenen Aufgaben unterschiedliche Verhaltensweisen zeigen, d.h. der Vorgang kann von Aufgabe zu Aufgabe differieren. Daher ist es angebracht, diese Vorgänge zu untersuchen, soweit wie möglich zu strukturieren sowie darauf basierende Schlüsse und entsprechende Konsequenzen bei der Gestaltung der Lernmaterialien zu ziehen. So werden kartographische Darstellungen 
vor allem im Hinblick auf den pädagogischen Wert und ihre Eignung für die verschiedenen Lerntypen betrachtet.

Dabei ermöglichen die Verwendung und Verflechtung diverser multimedialer Elemente und kartographischer Ausdrucksformen Benutzern (Schülern) mit unterschiedlichem Lernverhalten, Geophänomene in individueller Art zu verstehen und ein so entstandenes Basiswissen (Lehrziel) entsprechend ihren persönlichen Interessen in selbständiger Arbeit zu erweitern.

Ein besseres Eingehen auf die Lernenden, die als Geographie-Lehrmittel benutzerangepasste Multimediale Kartographische Atlas-Informationssysteme benützen, kann durch Anwendung lernpsychologischer Grundlagen in Verbindung mit neuen Technologien erfolgen, da für die Informationserfassung mehrere Interaktionsmöglichkeiten zur Verfügung gestellt werden. Diese Intentionen werden in weiterer Folge dargestellt.

\section{Wahrnehmungsverlauf und lernpsychologische Grundlagen}

Die Aufnahme und Verarbeitung von Information ist ein komplexer Prozess. Information wird gesammelt, organisiert, aufgenommen und verarbeitet, mit schon vorhandenem Wissen verglichen und dann im Gedächtnis verankert (vgl. Abb. 1). Dieser Verlauf ist zwar prinzipiell bei allen Menschen ident, dennoch sind im Detail Abweichungen feststellbar. So können verschiedene Wahrnehmungstypen erkannt werden. Es wird davon ausgegangen, dass es Kanalpräferenzen, Vorlieben hinsichtlich der Herangehensweise an neue Information, bevorzugte Verwendung einer Gehirnhälfte und einen unterschiedlichen Aktivitätsgrad bei der Informationssuche gibt. Aus diesen Präferenzen und Strategien können Lernstile und Lerntypen erkannt und definiert werden. Diese Differenzierungen dienen als Grundlage bei der Darbietung von Stoffinhalten und bei der Gestaltung bzw. Anwendung der Lernmaterialien, um eine Benutzergruppenanpassung zu erzielen.

Weiters werden die Grundlagen der Aufnahme und Verarbeitung von Information im Hinblick auf Informationserschließung durch kartographische Kommunikation besprochen. Die angesprochenen Lernstile und Lerntypen existieren in einer weitaus ausführlicheren Form und in einer größeren Vielfalt als in dieser Arbeit beschrieben wird. Hier werden nur jene Präferenzen berücksichtigt, die sowohl durch Literatur und Studien eindeutig belegt werden als auch für kartographische Fragestellungen relevant erscheinen und die in einem Interaktiven Multimedialen Kartographischen Informationssystem berücksichtigt werden können.

\subsection{Informationssuche}

Unter Informationssuche versteht man den Zugang eines Lernenden zu Informationen, deren Selektion in eine bestimmte Richtung und unter Präferenz eines bestimmten Sinnesorganes vorgenommen wird (vgl. Abb. 1). 
294 Beatrix Brunner-Friedrich, Mirjanka Lechthaler und Eszter Simonné-Dombóvári

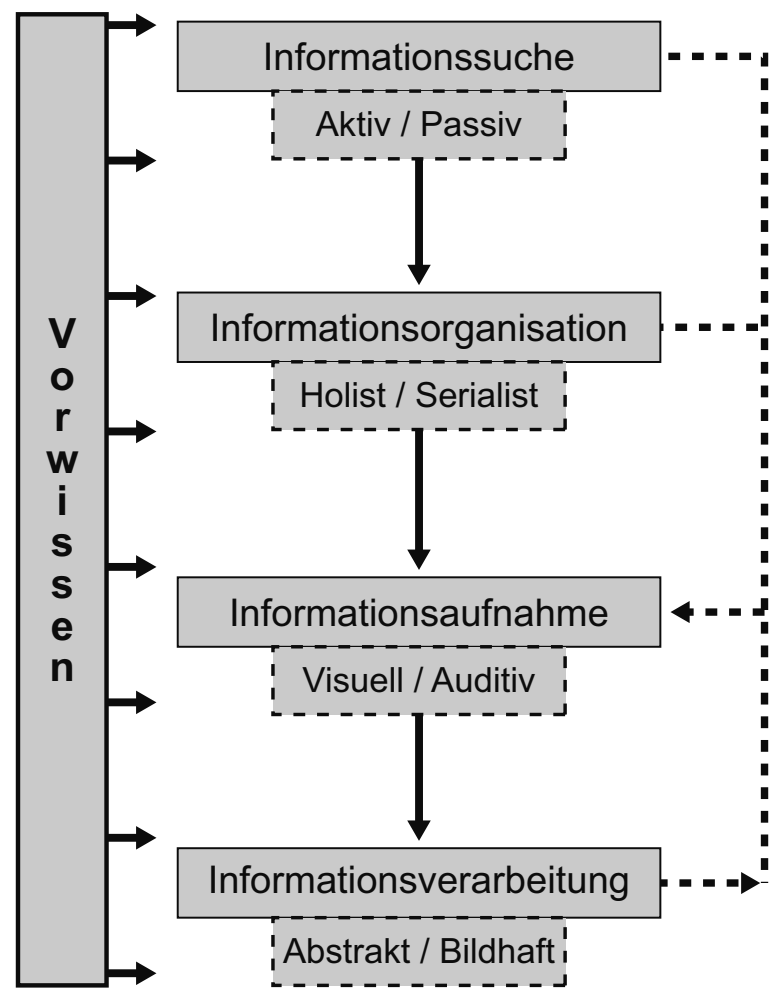

Abb. 1: Wahrnehmungsverlauf mit Ansätzen für eine Benutzergruppenanpassung (BRUNNER-FRIEDRICH 2004a)

\subsection{Informationsorganisation}

Bei der Informationsorganisation (auch Lernstrategie) ist der Grad der Detailorientierung, mit der ein Lernender an eine Information herangeht, das wesentliche Unterscheidungsmerkmal. Das Pask-Modell (JonAssen \& Grabowski 1993) geht von einem dualistischen Ansatz aus und unterscheidet zwischen Serialisten, die stufenweise von Konkretionen zu Abstraktionen gelangen, und Holisten, die laufend zwischen Konkretionen und Abstraktionen interferieren. Während sich der Holist auf allgemeine und globale Beziehungen konzentriert, stellt der Serialist kleine Zusammenhänge und Einzelheiten in den Mittelpunkt. Holisten (Globale Typen) sind stärker global orientiert und betrachten meist mehrere Aspekte gleichzeitig, sie gehen von einem Gesamtbild hin zu Details. Serialisten (Sequentielle Typen) konzentrieren sich zunächst auf Details, erst später wird ein allgemeines Konzept aufgebaut (vgl. Abb. 1). 


\subsection{Informationsaufnahme - Lernen}

Die Informationsaufnahme kann auf zwei Arten erfolgen. Es wird unterschieden zwischen mechanischem und sinnvollem Lernen. Bei mechanischem Lernen wird die Information wortwörtlich und nicht inhaltlich gelernt, sie wird nicht auf das Vorwissen bezogen. Wird die Information zufallsfrei und inhaltlich auf vorhandene Wissens- und Wertestrukturen bezogen, erfolgt eine Verankerung des neuen Lernstoffes in bereits vorhandene Strukturen. Man spricht vom sinnvollen Lernen (MAYER 2000).

Weiters wird nach EDELMANN (1996) zwischen rezeptivem und explorativ-entdeckendem Lernen unterschieden. Bei rezeptivem Lernen wird dem Schüler der vollständige Lerninhalt in seiner fertigen Form übermittelt. Es werden keine selbstständigen Entdeckungen verlangt, der Lernstoff wird nicht mit dem Vorwissen assimiliert, sondern auswendig gelernt, sodass er zu einem späteren Zeitpunkt zur Verfügung steht oder reproduziert werden kann. Das wesentlichste Merkmal des entdeckenden Lernens ist die Tatsache, dass der Hauptinhalt dessen, was gelernt werden soll, nicht gegeben ist, sondern vom Schüler entdeckt werden muss und in bereits vorhandene Strukturen verankert wird.

Durch die Kombination dieser Lernformen entstehen vier Grundformen. Im Folgenden werden aber nur zwei dieser Formen weiter verwendet: sinnvolles, exploratives (entdeckendes) Lernen, im Weiteren als Aktives Lernen bezeichnet, und sinnvolles, rezeptives Lernen, hier als Passives Lernen bezeichnet.

Ein weiterer Unterschied bei der Informationsaufnahme (auch im Lernstil) ist die Kanalpräferenz, d.h. mit welchem Sinneskanal eine Information bevorzugt wahrgenommen wird. Aus der Kognitionspsychologie ist seit längerem bekannt, dass menschliches Erinnerungsvermögen um bis zu 90\% gesteigert werden kann, wenn Methoden kombiniert werden: sehen und hören, selbst sagen, selbst tun. Die unterschiedlichen Lernstile werden auch als Lerntypen bezeichnet. Der visuelle Typ lernt durch Lesen und mithilfe von Bildern, visuelle Unordnung stört ihn. Der auditive Typ nimmt Information bevorzugt durch Hören auf. Plötzliche Geräusche führen bei ihm zu einer Ablenkung (Feichtenberger 2001) (vgl. Abb. 1).

\subsection{Informationsverarbeitung - Lernen}

In einem letzten Schritt wird die aufgenommene Information verarbeitet, mit schon vorhandenem Wissen verglichen und dann im Gedächtnis verankert. Dabei spielt einerseits das mechanische bzw. sinnvolle Lernen, andererseits aber auch die Art, wie die Information bevorzugt gespeichert wird - eher abstrakt oder eher bildhaft - eine Rolle. Diese präferierte Verarbeitung spiegelt sich auch in der Informationsaufnahme wider, d.h. ein abstrakt denkender Lerner bevorzugt auch abstrakt präsentierte Information (vgl. Abb. 1). 


\section{Einsatz interaktiver und multimedialer kartographischer Informationssysteme als Geographie-Lehrmittel}

Erfolgreiches Lernen hängt von einem guten Unterricht und der Fähigkeit, Wissen zu speichern sowie davon ab, wie die Schüler beim Lernen vorgehen. Schüler, die $\mathrm{zu}$ einem selbstregulierten Lernen in der Lage sind, erzielen demnach auch bessere Leistungen in der Schule. Der Unterricht soll Schüler auf diese Verhaltensweise vorbereiten, so dass sie auch für das spätere Berufsleben eine wirksame selbstgesteuerte Arbeitsweise erlernen.

\subsection{Neue Rolle des Lehrers in der veränderten Arbeitsweise der Schüler}

Voraussetzung für ein selbstreguliertes Lernen ist allerdings eine hohe Motivation. Diese positive Einstellung ist nach DÖRR \& STRITTMATTER (2002) zu erreichen, indem Erwartungen provoziert werden, die Lernen auslösen. Durch den Einsatz von verschiedenen Medien und von Multimedia wird die Motivation gesteigert. Die Verwendung von Computern im Unterricht wirkt sich positiv auf die Motivation der Schüler aus und unterstützt den Individualisierungsprozess in der Kognition sowie das selbstgesteuerte Lernen im Hinblick auf Tempo und persönlichen Zugang zum Lernstoff. Der Lehrer kann dabei jeden Schüler seinen Eigenheiten entsprechend fördern, ohne gleichzeitig die anderen in ihrem Lernfortschritt zu behindern. Diese selbstständige Arbeitsweise ermöglicht, dass Schüler Wissen nicht nur reproduzieren, sondern auch konstruieren (DÖRR \& STRITTMATTER 2002).

Die Veränderung der Arbeitsweise der Schüler bedingt eine neue Rolle des Lehrers (DeHMER \& Koller 1997). Bei der Forderung nach selbstständigem Arbeiten der Schüler kann der Lehrer nicht als reiner Wissensvermittler tätig sein. Er muss von dieser Rolle abweichen und zumindest für manche Aufgaben ein Coach sein. Bei der selbständigen Arbeitsweise erarbeitet der Schüler bis zu einem gewissen Grad das Stoffgebiet eigenständig (BRUnNER-Friedrich 2004b; Simonné-DombóvÁRi 2009). Ob er das nun mit einem Tutorial, das der passiven Strategie entgegenkommt, vornimmt oder mit anderen Mitteln, bleibt ihm selbst überlassen. Er muss in dieser Frage also eigenständig entscheiden. Diese Entscheidung fördert das selbstregulierte oder selbstgesteuerte Lernen.

Andererseits heißt unselbständige Arbeitsweise, dass der Schüler das gesamte Stoffgebiet vom Lehrer vorgetragen bekommt. Der Unterrichtende erklärt, stellt Fragen und Aufgaben und gibt konkrete Hinweise im Frontalunterricht.

Es ist allerdings offensichtlich, dass ein passiver Lerntyp eher eine unselbstständige und der aktive eine selbstständige Arbeitsweise bevorzugt.

\subsection{Medien im Unterricht}

Für den Begriff Medien findet man unterschiedliche Definitionen, welche auch Diskrepanzen erkennen lassen (BRUNNER-FrIEDRICH 2004b). Nach MüLLER \& DRANSCH 
(2001) dienen Medien innerhalb eines Kommunikationsprozesses als technische Geräte zur Informationsspeicherung und -weitergabe. Sie sind Informationsträger (Buch, Tafel, Dia-, Overheadprojektor, Beamer, Videoanlage, Bildschirm, etc.), wobei WeIDENMANN (2002) an die Codierung der Information denkt.

Nach beiden oben angeführten Erklärungsansätzen erscheint es sinnvoll, Medien als eine Kombination aus drei Komponenten zu sehen: Codierung, Träger, Gerät. Von Multimedia im Sinne der drei Komponenten kann dann gesprochen werden, wenn zwei oder mehrere Medien kombiniert werden, wobei sich diese entweder durch den Träger oder durch die Codierung unterscheiden (BRUNNER-FRIEDRICH 2004b).

In den vorliegenden Arbeiten wird dieser Ansatz verwendet, wobei gilt, dass multimediales Lernen nach MAYER (2000) jederzeit, an jedem beliebigen Ort und individuell angeboten und durchgeführt werden kann. Je besser die Unterrichtsmedien an die individuellen Bedürfnisse des Einzelnen angepasst sind, desto größer wird der Lernerfolg vor allem durch leichteres Behalten sein.

\subsubsection{Computer im Unterricht}

Der Computer kann im Unterricht auf unterschiedliche Weise eingesetzt werden. Generell erstreckt sich das Spektrum von schulbuchergänzender bis zu fast schulbuchersetzender Software auf der einen Seite und von extensiver bis zu intensiver Internetnutzung bei der Arbeit auf der anderen Seite (RINSChEDE 2005). Sowohl das Internet mit verschiedenen Online-Lernsoftwareprogrammen als auch CD-ROMs sind gut geeignet, um eine Fülle von Informationen mithilfe von unterschiedlichen Fragestellungen in selbstständiger Weise zu bearbeiten (SITTE 2001a). Ein aktueller Überblick über die österreichische Unterrichtssoftware für Geographie und Wirtschaftskunde ist im schulgeographischen Internetportal Österreichs unter Education Highway im Innovationszentrum für Schule und neue Technologie zu finden [1].

\subsection{Vorteile und Nachteile der neuen Medien}

Doch der Einsatz von neuen Medien bringt nicht nur Vorteile mit sich. Auf der einen Seite steigt die verfügbare Informationsmenge immer mehr an, auf der anderen Seite sind die Rezeptions- und Verarbeitungskapazität des Benutzers und die ihm zur Verfügung stehende Zeit begrenzt (KolLER 2001). Ein Schüler wird die Auswahl nicht nur nach sachlichen und ethischen Aspekten vornehmen, er wird auch das wählen, was ihm unterhaltsam und attraktiv erscheint. Nach Postman (1985) lernen Kinder, unter anderem durch Fernsehen, dass Lernen, also Informationsverarbeitung, Unterhaltung bedeutet. Sie verlernen aber gleichzeitig, dass Ausdauer und aktives Erarbeiten ebenso wichtig sind. Es wird vielmehr zufällig und nebenbei gelernt. Das passende Schlagwort zu dieser Einstellung lautet edutainment (BRUNNER-FRIEDRICH 2004b). Hinter diesem Ausdruck verbirgt sich folgendes Motto: „Ich lerne, weil (es) mir Spaß macht - ich lerne aber auch nur, was mir Spaß macht!“ Auch das Verhalten bei der Suche nach neuer Information ist verändert. Information wird nicht mehr zielgerichtet gesucht, sie ergibt sich bei Unterhaltung und Zeitvertreib. Das Suchen wird zum Selbstzweck. 
Für die zielgerechte Informationsauswahl ist eine Unterstützung durch den Lehrer daher unumgänglich.

Neue Medien erwecken anfangs immer den Anschein, Lehren und Lernen wesentlich verbessern zu können. Vergleichsuntersuchungen zeigen zunächst auch eine Überlegenheit der neuen Medien. Dieser Erfolg wird aber meist dadurch bedingt, dass neue Medien auch eine neue Unterrichtsmethode hervorbringen müssen (DöRR \& STRITTMATTER 2002).

Der Einsatz von verschiedenen Medien, von Multimedia und speziell des Computers erscheint durchaus sinnvoll. Er steigert die Motivation der Schüler (OECD 2003). Allerdings darf die Verwendung von unterschiedlichen Medien und Multimedia nicht nur um ihrer selbst Willen geschehen. Sie müssen vielmehr didaktisch sinnvoll in verschiedenen Unterrichtsformen (Frontalunterricht, offenes Lernen, Projektarbeit), verbunden mit neuen Methoden eingesetzt und bei der Arbeit durch den Lehrer zielführend unterstützt werden (BRUNNER-FRIEDRICH 2004b).

\subsection{Interaktives Multimediales Atlas-Informationssystem}

Ein Teil des Unterrichts in Geographie und Wirtschaftskunde (GW) basiert auf der Arbeit mit einem Schulatlas ([2], [3]) - vor allem auf dem Umgang mit Karten und kartenverwandten Darstellungen sowie daraus erworbenen raumbezogenen Informationen. Dazu werden interaktive und den neuen Medien entsprechend aufbereitete Lerninhalte zur Verfügung gestellt.

Über neue (elektronische) Medien im Unterricht auf diesem Gebiet gibt es eine Fülle an fachdidaktischer analoger und Online-Literatur (ANDERSON et al. 2001; KolLER 2001; SitTE 2001b, 2004, [4], [5], [6]). Nicht alle im IT-Netz angebotenen Unterlagen und Ergänzungen wie CD-ROMs, DVDs und Web-Applikationen für Schüler und Lehrer entsprechen den didaktischen und inhaltlichen Anforderungen des GW-Unterrichts. Dazu sollen einige Begriffe definiert werden.

Ein Geographisches Informationssystem (GIS) ist nach MüLLER (2001) als Software zu verstehen, mit deren Hilfe Geodaten (Objektraumgeometrie und georeferenzierte Sachdaten) erfasst, verwaltet, analysiert und als Geoinformationen ausgegeben werden können.

Ein Kartographisches Informationssystem (KIS) ist ein Informationssystem, das aus Bildschirmkarten oder Karteninformationen besteht. Die raumbezogenen Daten, nämlich die primären Inputdaten (auch aus GIS-Anwendungen) und/oder vorliegende sekundäre Karteninformationen mit bereits maßstabsangepassten Objektvisualisierungen sind nach den Regeln der kartographischen Modellbildung, d.h. Datengeneralisierung, -harmoniserung und -visualisierung maßstabsgebunden, grafikdefiniert und dem Ausgabemedium angepasst verarbeitet, gespeichert und zum Aufruf bereit gehalten (BRUNNERFriedRich et al. 2006; LeChTHALER et al. 2006a, 2006b; ORTAG 2008). Jede beliebige Signaturgrafikfestlegung und -änderung verlangt unweigerlich nach kartographischen Generalisierungs- und Harmonisierungsmaßnahmen (KelnHofer 2004). Nur so wird ein störungsfreier Wahrnehmungs- und Kommunikationsprozess der raumbezogenen Daten durch Karteninformationen in einem Informationssystem ermöglicht. 
Ein Atlas-Informationssystem (AIS) ist „... eine auf nutzerorientierte Kommunikation ausgerichtete Sammlung raumbezogenen Wissens..." (Bollmann \& Koch 2001). Es ist ein KIS zur Informations- und Entscheidungsfindung, in welchem mithilfe des Datenmanagements (Speicherung, Verwaltung, Manipulation) und systemeigener Funktionen selektive Datenzugriffe, -verknüpfungen und systemgesteuerte restriktivflexible Datenvisualisierungen ermöglicht werden. Neben den Karteninformationen wird eine themenbezogene und -verlinkte Vielzahl an unterschiedlichen Informationsarten wie Grafiken, Diagrammen, Tabellen, Texten und Fotos zur Unterstützung der Kommunikation angeboten. Ein Interaktives Multimediales Atlas-Informationssystem (IMAIS) ist zumeist als Datenportal oder -schnittstelle (KeLnhofer 2004; LeChthaLeR et al. 2007) aufgebaut und dient der benutzerseitigen Informationsakquisition. Die Kommunikation erfolgt durch unterschiedliche, vom Systemdesigner vorgesehene Informationszugriffsmöglichkeiten auf Basis von interaktiven und kartographisch aufbereiteten Visualisierungen, welche zusätzlich durch themenangepasste multimediale Sequenzen unterstützt werden.

Allgemein sollte der Benutzer (Schüler) interaktiv sowohl auf alle raumbezogenen Sach-/Geometriedaten und -informationen, seiner Aufgabe angepasst, zugreifen, Analysen durchführen und bis zu einem gewissen Grad die Resultate seiner Abfragen auch visualisieren können (RAmos DA Silva \& CARTwright 2005). So kann beispielsweise die individuelle, interaktive Kartengestaltung in Bezug auf Symbol- und Farbwahl sowie die Anwendung bestimmter Gruppierungsverfahren im Bereich der thematischen Sachdatenumsetzung eine weitere Individualisierung oder Benutzergruppenanpassung darstellen. Das System führt den Benutzer in einem restriktiv-flexiblen Modus oder mit einem kontrollierten Zugriff zu den Bearbeitungs- und Visualisierungswerkzeugen und ermöglicht somit nur deren kartographisch sinnvollen Einsatz (Ormeling 2001; Spanring, Gartner \& Kriz 2005; Lechthaler, Spanring \& Katzlberger 2006a).

\section{Anpassung Kartographischer Informationssysteme an die Benutzergruppen}

Kartographische Informationssysteme basieren auf kartographischen Visualisierungen, die für Informationserschließung oder direkt für Analysen eingesetzt werden. Kartographische Visualisierungen sind eine abstrakte, generalisierte Abbildung der Wirklichkeit. Der Abstraktionsgrad ist einerseits maßstabsgebunden und hängt andererseits von den verwendeten Signaturen ab. Für räumlich differenzierte Phänomene sind Karten (und kartenverwandte Darstellungen) die beste Ausdrucksform und Texten weit überlegen.

Prinzipiell unterstützen Karten den abstrakt und visuell denkenden Lerntyp, wobei für einige Benutzer aufgrund der Abstraktion bei der Interpretation der Darstellungen Schwierigkeiten entstehen können. Da eine Karte auf unterschiedliche Arten gelesen werden kann, ist sie sowohl für Serialisten als auch für Holisten geeignet. 
Durch den Einsatz von Multimedia, Animationen, Simulationen und Interaktivität können kartographische Ausdrucksformen ausgewählt werden und kann man Inhalte entsprechend unterstützen, ergänzen und jedem Lerntyp, jedem Alter und jeder Schulstufe [2] auf unterschiedliche Weise (in verschiedener Codierung und Sinnesmodalität) angepasst präsentieren. Die Anpassung an Benutzergruppen erfolgt entweder durch den Weg durch das Informationssystem (aktiv/passiv, Holist/Serialist) oder durch die verwendeten Präsentationsformen (visuell/auditiv, abstrakt/bildhaft). Diese Benutzergruppenanpassung kann wie folgt dargestellt werden (BRUNNER-FRIEDRICH 2004b):

- Verwendung unterschiedlicher Zugänge zu den Informationen:

- vom Ganzen ins Detail / vom Detail ins Ganze $\rightarrow$ Holist / Serialist;

- Führung durch das System / eigenständiges Erforschen der Inhalte $\rightarrow$ passiv / aktiv;

- Verwendung unterschiedlicher Medien:

- View-only Karten / Interaktive Karten $\rightarrow$ passiv / aktiv;

- Karten / Erweiterung der Karten um akustische Elemente $\rightarrow$ visuell / auditiv;

- geschriebener / gesprochener Text $\rightarrow$ visuell / auditiv;

- Verwendung unterschiedlicher kartographischer Ausdrucksformen (schulstufenabhängig):

- Luftbild / Karte (Karte / Luftbild) $\rightarrow$ bildhaft / abstrakt (abstrakt / bildhaft)

- Verwendung unterschiedlicher Abstraktionsgrade der Kartenzeichen (schulstufenabhängig):

- bildhaft bis geometrisch-abstrakt $\rightarrow$ bildhaft bis abstrakt.

Die Anpassung an die Schulstufe ist besonders in allen Ausgaben des österreichischen Kozenn-Schulatlasses (AHS-Unter- und Oberstufe) zu sehen ([2], [3]). Im Vergleich zum umfangreichen und komplexen Neuen Kozenn-Atlas werden die Karten im Kozenn-Schulatlas [3] und im Hölzel-Atlas für die 5. bis 8. Schulstufe [2] grafisch mit kräftigen Farben oder bildhaften Signaturen und Bilderkarten an das Bildverständnis der 10- bis 14-Jährigen angepasst (SITTE 2001b).

\subsection{Interaktivität und Benutzergruppenanpassung im IMAIS}

Wie in der Einleitung erwähnt, orientiert sich der Unterricht im Allgemeinen an kognitiven und instrumentalen Lernzielen, welche von den Lernenden unterschiedliche Aktivitätsgrade verlangen. Der Schüler, der beim Lernen eine eher aktive Rolle einnimmt und die Information auch aktiv sucht und sammelt, will ein Thema eigenständig erarbeiten, um zu neuem Wissen zu kommen. Bei der Fragestellung navigiert der aktiv Lernende im IMAIS und wählt eigene Wege der Informationserschließung, z.B. indem er Orte/Gebiete mit sichtbarer Klimaverschiebung selbst zu finden sucht. Im Gegensatz dazu erwartet ein passiver Schüler, dass er in vorgefertigten Unterlagen, durch Vorträge oder per Knopfdruck im IMAIS relevante Informationen über das genannte Thema Klimaverschiebung erhält. Sollen diese Zugangsarten oder Lerntypen in einem IMAIS berücksichtigt und umgesetzt werden, so ergeben sich nach BRUNNER-FRIEDRICH (2004b) folgende Modi: 
-Vorführmodus: Lernen mit dem System bei geringer Interaktivität. Der Weg zum Erarbeiten ist vorgeschrieben. In diesem Modus sind dieselben Themenbereiche (mit Unterkapiteln) wie beim Forschungsmodus enthalten, der Schüler sucht nicht nach den Inhalten, sondern geht sie nach einer vom System vorgeschlagenen Reihenfolge durch. Ein ständig angezeigtes Inhaltsverzeichnis, in dem schon besuchte Themenbereiche farblich unterlegt sind, bietet einen Überblick.

- Forschungsmodus: Lernen mit dem System bei hohem Interaktivitätsgrad. Der Weg zum Erarbeiten ist vollkommen dem Benutzer überlassen. Der Schüler kann in frei gewählten Schritten Themenkomplexe bearbeiten, wobei er lediglich durch eine holistische oder eine serialistische Aufgabenstellung zu Arbeitsbeginn gelenkt wird.

- Lexikonmodus: Nachschlagen im System, Suchen von Definitionen und Informationen. Dieser Modus steht beiden Benutzergruppen in gleicher Weise zur Verfügung.

\section{Fazit}

Basierend auf lernpsychologischen, pädagogischen und kartographischen Grundlagen wurden Konzepte für den Einsatz Kartographischer Informationssysteme, insbesondere interaktiver und multimedialer Atlas-Informationssysteme als Hilfsmittel im Geographieunterricht in der Schule aufgezeigt.

Der Wahrnehmungs- und Lernverlauf ist zwar prinzipiell bei allen Menschen ident, dennoch sind im Detail Abweichungen feststellbar. So können verschiedene Lernstrategien, Lern- und Denktypen sowie Kognitionstypen erkannt werden. Bei der Darbietung von Stoffinhalten und bei der Gestaltung und Anwendung der Lernhilfen kann unter Berücksichtigung dieser Unterschiede eine Benutzergruppenanpassung erzielt werden.

Die Kartographie bietet viele Möglichkeiten, diesen Anforderungen gerecht zu werden. Dabei muss es das Ziel sein, kartographische Ausdrucksformen und Gestaltungsmittel sowie technische Gegebenheiten und Technologien so zu verwenden und zu kombinieren, dass bessere Lernresultate in Geographie und Wirtschaftskunde erreicht werden. ${ }^{1)}$

\section{Literaturverzeichnis}

Anderson J., Carriere J., Le Sann J. (2001), An Internet Atlas in the Schools of Quebec: Reality and Ideals. In: Proc. of $20^{\text {th }}$ Intern. Cartogr. Conf., Beijing, China, S. 2944-2951. Bollmann J., Косн W.-G. (2001), Lexikon der Kartographie und Geomatik. Heidelberg - Berlin, Spektrum Akad. Verlag.

\footnotetext{
1) Lernpsychologische und mediale Grundlagen im Hinblick auf Kartographische Informationssysteme als Geographie-Lehrmittel werden in den praktischen Anwendungen der IMAIS-Prototypen InMuAIS und GeoLearn im nächsten Band (Bd. 152) der MÖGG vorgestellt werden.
} 
BRUNNER-FrIEDRICH B . (2004a), Lerntypengerechter Schulatlas - Konzept eines benutzergruppenangepassten Kartographischen Informationssystems zur Präsentation raumbezogener Informationen. In: Strobl J., Blaschke T., Griesebner G. (Hrsg.), Angewandte Geoinformatik 2004, S. 51-56. Heidelberg, Wichmann.

BRUNNER-FrIEDRICH B. (2004b), InMuKIS - Konzept eines benutzergruppenangepassten Interaktiven Multimedialen Kartographischen Informationssystems für die Schule zur Präsentation raumbezogener Informationen. Wien, Techn. Univ. Wien, Inst. f. Geoinformation u. Kartographie, Diss.

Brunner-Friedrich B., Stadler A. (2006), Ansatz zur Formalisierung der Sachdatenumsetzung in einem interaktiven Atlas-Informations-System. In: Kriz K., CArtwright W., Pucher A., Kinberger M. (Hrsg.), Kartographie als Kommunikationsmedium, Cartography as a Communication Medium (= Wiener Schriften z. Geogr. u. Kartogr., 17), S. 142-150

Dehmer W., Koller A. (1997), Internet für Geographielehrer - leicht gemacht! In: Geographie heute, 152, S. 36-38.

Dörr G., Strittmatter P. (2002), Multimedia aus pädagogischer Sicht. In: Issing L.J., KLimSA P. (Hrsg.), Information und Lernen mit Multimedia (3. Aufl.), S. 28-42. Weinheim, Psychologie-Verlags-Union.

Edelmann W. (1996), Lernpsychologie. 5. vollst. überarb. Aufl. Weinheim, PsychologieVerlags-Union.

Feichtenberger C. (2001), Lerntypengerechter Fremdsprachenunterricht mit Suggestopädie. Universität Graz. - http://www.semigator.de/trainer/profil/145226/Dr-Claudia-Feichtenberger

Jonassen D.H., Grabowski B.L. (1993), Handbook of Individual Differences, Learning and Instruction. Hillsdale, N.J., Lawrence Erlbaum Associates.

Kelnhofer F. (2004), Mediale Dichotomie in der thematischen Kartographie, eine notwendige Konsequenz „moderner Kartographie“? In: Kainz W., Kriz K., Riedl A. (Hrsg.), Aspekte der Kartographie im Wandel der Zeit (= Wiener Schriften z. Geogr. u. Kartogr., 16), S. 197-204.

Koller A. (2001), Computereinsatz und Online-Medien im GW-Unterricht. In: Sitte W., Wohlschlägl H. (Hrsg.), Beiträge zur Didaktik des Geographie- und WirtschaftskundeUnterrichts (= Materialien z. Didaktik d. Geogr. u. Wirtschaftskunde, 16), S. 59-75.

Lechthaler M., Spanring C., Katzlberger G. (2006a), Cartographic Information System “ÖROK Atlas Online" - AIS Austria as a Communication Portal of Austrian Basic Geo-Data. In: Geodetski list, 60, 4, S. 241-257.

Lechthaler M., Stadler A. (2006b), Cross Media gerechte Kartengraphik in einem IS. In: Schrenk M. (Hrsg.), CORP 2006 - 11. Internationale Konferenz zu Stadtplanung und Regionalentwicklung in der Informationsgesellschaft, S. 443-452. Wien.

Lechthaler M., Strasser M., Todor R. (2007), Die Rolle der Kartographie in räumlichen Entscheidungsprozessen. In: Mitt. d. Österr. Geogr. Ges., 149, S. 285-304.

MAYer H. (2000), Einführung in die Wahrnehmungs-, Lern- und Werbe-Psychologie. München - Wien - Oldenburg, Oldenburg Wissenschaftsverlag.

Müller A. (2001), Geoinformationssystem. In: Bollmann J., Koch W.-G. (Hrsg.), Lexikon der Kartographie und Geomatik, Bd. 1, S. 304-306. Heidelberg - Berlin, Spektrum Akad. Verlag.

Müller A., Dransch D. (2001), Medien. In: Bollmann J., Koch W.-G. (Hrsg.), Lexikon der Kartographie und Geomatik, Bd. 2, S. 134-136. Heidelberg - Berlin, Spektrum Akad. Verlag.

OECD (2003), Schülermotivation entscheidend für den Lernerfolg. Fazit einer neuen OECD Studie. 
Lernpsychologische und mediale Grundlagen im Hinblick auf KIS als Geographie-Lehrmittel 303

ORMELING F. (2001), Das Bändigen von Multimedia-Konzepten für den Online-Atlas der Niederlande. In: HerRmann Ch., Asche H. (Hrsg.), Web.Mapping 1 - Raumbezogene Information und Kommunikation im Internet, S. 178-189. Heidelberg, Wichmann.

ORTAG F. (2008), Präsentationsformen in der Fußgängernavigation. Sprachausgabe versus Kartendarstellung - ein Feldexperiment. Saarbrücken, VDM Verlag Dr. Müller.

Postman N. (1985), Wir amüsieren uns zu Tode. Frankfurt am Main, Fischer.

Ramos da Silva C., CARTwright W. (2005), Atlases from paper to digital medium. In: STefanikis E., Peterson M.-P., Armecanis C., Delis V. (Hrsg.), Geo-Hypermedia'05, Proc. of the $1^{\text {st }}$ Intern. Workshop on Geographic Hypermedia. Denver, Colorado, U.S.A.

Rinschede G. (2005), Geographiedidaktik. Paderborn, Ferdinand Schöningh.

Simonné-DombóváRi E. (2009), Möglichkeiten der Kartenlernspiele im Geographieunterricht (für die 4., 6. und 8. Klasse Gymnasium), Development of mapgames in the geographic education (for $4^{\text {th }}, 6^{\text {th }}$ and $8^{\text {th }}$ grade High Schools pupils). Budapest, Eötvös Loránd Univ., Lehrstuhl f. Kartogr. u. Geoinformatik, Diss.; Manus.

SitTe Ch. (2001a), Einflussfaktoren auf den Wandel des Schulfaches. In: Einführung in die Didaktik der Geographie und Wirtschaftskunde. Skriptum. Wien, Inst. f. Geogr. u. Regionalforschung d. Univ. Wien.

SitTe Ch. (2004), Wie „politisch“ ist Geographie und Wirtschaftskunde? Eine Analyse im Zusammenhang mit neuen Oberstufen-Lehrplänen. In: GW-Unterricht, 93, S. 40-49.

Sitte W. (2001b), Schulatlas I (Zu seiner Geschichte in Österreich). In: Sitte W., WohlschlägL H. (Hrsg.), Beiträge zur Didaktik des Geographie- und Wirtschaftskunde-Unterrichts (= Materialien z. Didaktik d. Geogr. u. Wirtschaftskunde, 16), S. 410-423. Wien.

Spanring Ch., Gartner G., Kriz K. (2005), ÖROK Atlas Online (Atlasinformationssystem Austria). In: Schrenk M. (Hrsg.), Real Models - Unreal World, CORP 2005 - GeoMultimedia 05, CD-Ausgabe, Wien.

Weidenmann B. (2002), Multicodierung und Multimodalität im Lernprozess. In: Issing L.J., Klimsa P. (Hrsg.), Information und Lernen mit Multimedia (3. Aufl.), S. 45-62. Weinheim, Psychologie-Verlags-Union.

URL [1] http://gw.eduhi.at/softlist.htm (besucht am 7.9.2009)

URL [2] http://www.hoelzel.at/prod/prod_nm/n_media.html (besucht am 7.9.2009)

URL [3] http://www.hoelzel.at/kozenn (besucht am 7.9.2009)

URL [4] http://www.bmukk.gv.at/schulen/unterricht/schulbuch (besucht am 7.3.2009)

URL [5] http://www.geoinfosystem.delfdidakt (besucht am 7.9.2009)

URL [6] http://homepage.univie.ac.at/Christian.Sitte/FD (besucht am 7.9.2009) 
